\title{
Implementation of a voltage sag characterization based on a DSP platform
}

\author{
A. Ortiz, M. Mañana, F. Ortiz, C. J. Renedo, F. Delgado, L. I. Eguíluz \\ Department of Electrical and Energy Engineering \\ E.T.S.I.I., Cantabria University \\ Avenida de Castros, 39005 Santander (Spain) \\ Phone: +34-942-201376, Fax: +34-942-201385 \\ E-mail: ortizfa@unican.es
}

\begin{abstract}
.
Voltage sags are nowadays the first power quality problem in the distribution power system. This work evaluates the real implementation of a voltage sag characterization based on a DSP platform. Several classification methods for voltage sags have been presented in the literature. Here the Bollen's classification is tested. Automation of sag assessment is desirable because manual analysis may be quite difficult. Thus, the fuzzy logic technique is chosen for this aim. This logic system generalizes the classical two-valued logic for reasoning under uncertainty. The fuzzy algorithm was first tested by simulation and then it was translated into $\mathrm{C}$ code in order to be implemented in a DSP based measurement platform, obtaining a satisfactory result.
\end{abstract}

\section{Key words}

Power quality, Voltage sags, Fuzzy Logic, Digital Signal Processing.

\section{Introduction}

In the new open-access and competitive power market, electricity consumers are in a unique position to demand a higher quality of service. The utilities or other power providers have to ensure a high quality of their service to remain competitive and retain/attract the customers. Efficient power quality (PQ) monitoring and analysis system can help to achieve this goal.

PQ monitoring can usually be a complex task involving hardware instrumentation and software packages. The instrumentation entails communication software to accomplish the expected functionality. Various types of intelligent electronic devices can be used for collecting the desired PQ data. Examples include dedicated digital fault recorders and protective relays [1-5].
After PQ data of interest are obtained, a comprehensive PQ assessment can be carried out depending on the purpose of the study. This may include power system and equipment modeling verification, PQ problem mitigation and optimization, and data analysis [4,5]. In most cases, automated PQ assessment is desirable because manual analysis may be difficult to carry out due to lack of time and special expertise. Specialized software tools can make use of intelligent techniques to automate the PQ assessment for improved accuracy and efficiency [5]. This paper is focusing on automated detection and classification of voltage sags that may facilitate the overall PQ assessment. Intelligent techniques like fuzzy logic or neural networks have been utilized for developing PQ tools. Thus, a fuzzy approach will be undertaken in our case.

For many applications, the accuracy and efficiency resulting from the existing approaches may not be adequate. Improved accuracy is desirable. Aiming at this goal, this paper proposes a fuzzy system for making a decision based on the features extracted from voltage measurements.

One of the typical problems in electrical distribution systems are voltage sags. These have a great incidence on the electrical equipment connected to the network in the same area. The sags cause more damages than the rest of disturbances because these have a greater frequency of occurrence.

During sags the voltage suffers a sudden reduction between $90 \%$ and $10 \%$. Immediately after a short period of time the voltage recovers its value. Conventionally the duration of voltage sag are between 10 milliseconds and 1 minute. The voltage sag depth is defined as the difference between the minimum effective value (RMS) during the sag and the reference voltage. Reductions which do not overtake the limit of the $10 \%$ will not be considered as sags. 
Nowadays the research in this issue is still getting new results. Thus, we will show here another step forwards in order to implement a fuzzy sag classification based on a DSP measurement platform.

\section{Causes of voltage sags}

Shortcircuits and Faults to ground.

The most common cause of voltage sags are shortcircuits and faults to ground. These cases lead to the more severe voltage falls and to most of the failures in equipment.

One example of voltage sag due to fault is shown below.

The case of Fig. 1 is associated with different voltage reductions in two phases while the rms value of the other one is not affected by the perturbation. Different effective values in the three phases are a common characteristic in voltage sags caused by faults. Only shortcircuits in the

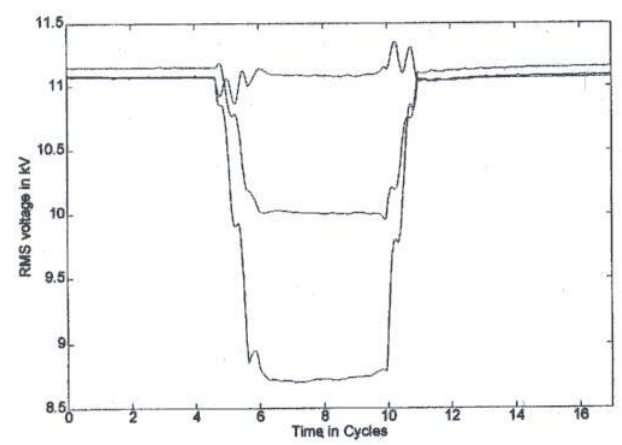

three phases lead to equal values. Another remarkable feature of these cases is the abrupt fall and recovery of the voltage; associated with the initiation and the compensation, respectively.

Fig.1 Voltage sag due to a non symmetrical fault.

\section{Motors and Transformers.}

Starting current of great induction motors is also a cause of voltage sags in power systems. The duration of this type of disturbance is much longer. Voltage sags due to a motor start show a sudden reduction with a slow recovery depending on ratio torque-speed of the load. Since induction motors are a balanced load, the reduction of the rms voltage is the same for the three phases.

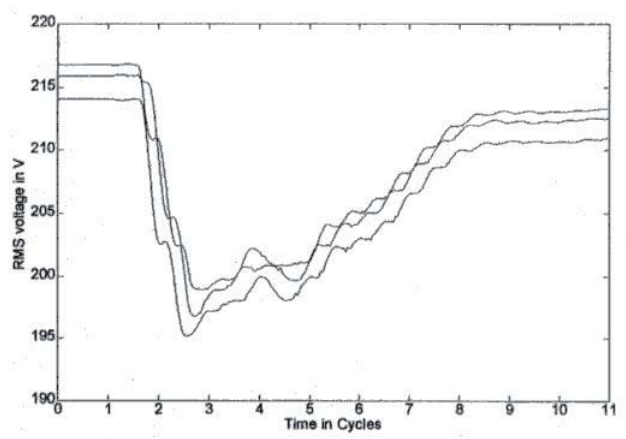

Fig.2 Voltage sag due to a motor starting
Transformers can also provoke voltage sags when they start. The process is characterized by a repetition of short sags with duration of a few milliseconds. These type of events have a great harmonic content. The voltage recovery it is quite slow and it depends on the decomposition of the dc component in the magnetic flux. Different effective values in the three phases are also a common characteristic in voltage sags caused by transformers

Post-Faults.

A fault is a very severe disturbance of the power system,

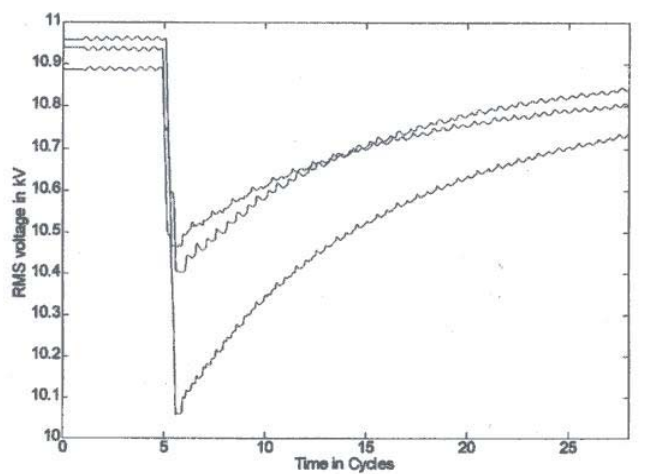

affecting as much the distribution as the load, and frequently at large distances. However, in this case the voltage recovery is not complete and does not reach the pre-fault value. The two main causes of Post-Faults are induction motors accelerations and transformers saturation.

Fig.3 Voltage sag due to a transformer starting

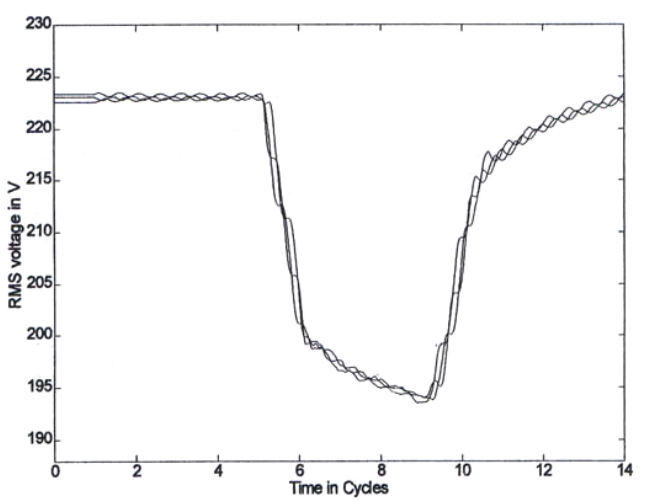

Fig.4 Voltage sag due to a three phase fault

During faults induction motors reduce speed, increasing gradually the current absorbed from the distribution system and causing a deep voltage reduction. These postfaults currents provoke post-faults voltage sags.

\section{Classification of voltage sags}

There are several classification methods for voltage sags:

- Classification using a magnitude.

- Classification using a magnitude and a time period.

- Classification using several magnitudes and a time period. 
- Bollen's classification.

In this work the Bollen's classification is used [6]. Seven types of sags are defined which correspond to the possible shortcircuits in the three-phase network and to the sag propagation through transformers. The most common types are A, B, C and D. Their characteristic voltage, by phase, correspond to the following expressions, Table I:

TABLE I.- Characteristic voltage for sags A, B,C and D.

\begin{tabular}{|c|c|}
\hline Type 'A' & Type 'B' \\
\hline$V_{a}=V$ & $V_{a}=V$ \\
$V_{b}=-\frac{1}{2} V-j \frac{\sqrt{3}}{2} V$ & $V_{b}=-\frac{1}{2}-j \frac{\sqrt{3}}{2}$ \\
$V_{c}=-\frac{1}{2} V+j \frac{\sqrt{3}}{2} V$ & $V_{c}=-\frac{1}{2}+j \frac{\sqrt{3}}{2}$ \\
\hline Type 'C' & Type 'D' \\
\hline$V_{a}=1$ & $V_{a}=V$ \\
$V_{b}=-\frac{1}{2}-j \frac{\sqrt{3}}{2} V$ & $V_{b}=-\frac{1}{2} V-j \frac{\sqrt{3}}{2}$ \\
$V_{c}=-\frac{1}{2}+j \frac{\sqrt{3}}{2} V$ & $V_{c}=-\frac{1}{2} V+j \frac{\sqrt{3}}{2}$ \\
\end{tabular}

\section{Fuzzy Logic}

This section briefly presents basic concepts of the fuzzy logic.

\section{Introduction to fuzzy system}

Fuzzy logic refers to a logic system that generalizes the classical two-valued logic for reasoning under uncertainty.

It is motivated by observing that human reasoning can utilize concepts and knowledge that do not have well defined or sharp boundaries [7].

Unlike the classical Boolean set allowing only 0 or 1 value, the fuzzy set is a set with a smooth boundary allowing partial membership. The degree of membership in a set is expressed by a number between 0 and 1 with 0 indicating entirely not in the set, 1 indicating completely in the set and a number in between meaning partially in the set. In this way, a smooth and gradual transition from the regions outside the set to those in the set can be described. A fuzzy set can thus be defined by a function that maps objects in the domain of concern (i.e. the universe of discourse) to their membership values in the set. Such a function is called the membership function. The two most widely used membership functions are the triangular and trapezoidal functions.

A fuzzy-expert system is an expert system that uses a collection of fuzzy sets and rules, instead of Boolean sets for reasoning about data. The rule in the fuzzy-expert system usually takes the form: If $\mathrm{A}$ is low and $\mathrm{B}$ is high; then $\mathrm{C}=$ medium. Where $\mathrm{A}$ and $\mathrm{B}$ are input variables, $\mathrm{C}$ is the output variable, and low, high and medium are membership functions defined for $\mathrm{A} ; \mathrm{B}$ and $\mathrm{C}$; respectively. The antecedent (the rule's premise) describes the degree that the rule applies, while the conclusion (the rule's consequent) assigns a membership function to the output variable. The set of rules in a fuzzy-expert system is known as the rule base or knowledge base. The computation of the output variable usually takes the following steps [7].

\section{Fuzzification.}

This step is also called Fuzzy Matching, which calculates the degree that the input data match the conditions of the fuzzy rules.

\section{Inference.}

Calculate the fuzzy set of the rule's conclusion based on its matching degree. There are two common approaches for the inference, namely the clipping method and the scaling method. Both methods generate the conclusion by suppressing the membership function of the consequent. The extent to which they suppress the membership function depends on the degree to which the rule is matched. The lower the matching degree, the more severe the suppression of the membership functions. The clipping method cuts off the top of the membership function, whose value is higher than the matching degree. The scaling method scales down the membership function in proportion to the matching degree. The scaling method is used here.

\section{Composition.}

Because a fuzzy rule-based system consists of a set of fuzzy rules with partially overlapping conditions, a particular input to the system often 'triggers' multiple fuzzy rules (i.e. more than one rule will match the input to a nonzero degree). Therefore, the composition is needed to combine the inference results of all the triggered rules to form a single fuzzy subset for the output variable.

The fuzzy disjunction operator Max is commonly used for constructing the output fuzzy set by taking the pointwise maximum over all the fuzzy subsets generated from the inference step, and is used here.

\section{Defuzzification.}

This step is to convert the fuzzy set of the output variable to a crisp number. Among the various types of defuzzification methods, the Center of Area (COA, or Centroid) and Maximum are the two most widely used techniques. The COA derives the crisp number by calculating the weighted average of the output fuzzy set while the Maximum method chooses the value with maximum membership degree as the crisp number. The maximum method is used here.

\section{Fuzzy System Proposed}

In order to propose a fuzzy algorithm for detection and classification of voltage sags, the voltage measurements resulting from the PCC should be analyzed. Thus, this section follows the same steps explained in the previous part, namely Fuzzification, Inference, Composition and 
Defuzzification. The interface of the Matlab Fuzzy Toolbox illustrates the main points of the process.

First of all, the number of inputs should be known. The system has six inputs corresponding to the three voltages measured, amplitude and angle.

The following action is to choose the membership function or functions. Then, it is possible to distinguish which parts of the inputs would define disturbances. The function chosen is trapezoidal, since it is a easy way to limit the inputs fuzzy sets.

For a better understanding of the idea some pictures will show every input fuzzification. For the case of the voltage amplitudes the fuzzy sets are equals for each of them. However, voltages shifts have specific details for the three phases.

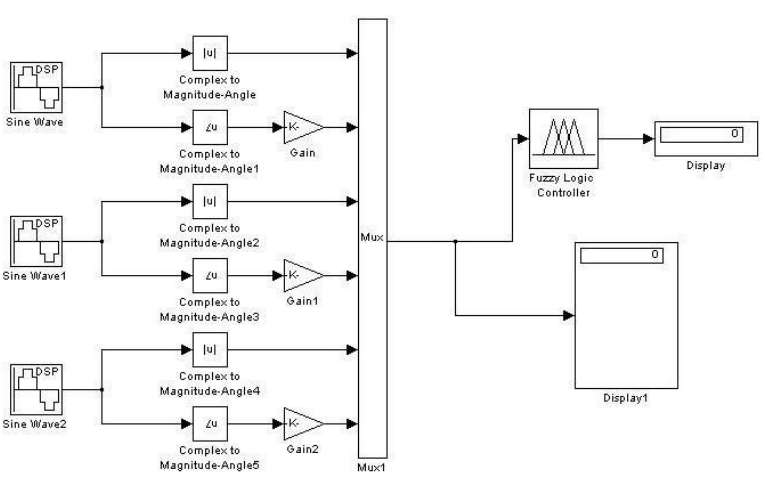

Fig.5 Fuzzy simulation using Matlab/Simulink

The three fuzzy sets proposed for the voltage amplitude are: the first one will define the normal operation (N.O.), the second one will detect the Sag and the third one will register the outage in the system (O.).

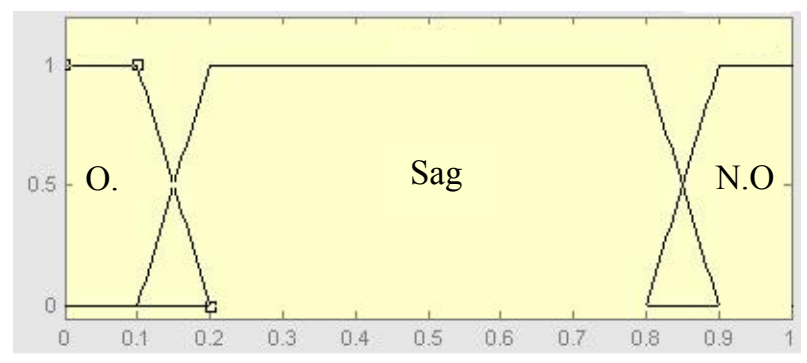

Fig. 6. Voltage amplitude fuzzy sets.

In this point, before considering the fuzzy sets for voltage shifts, the variation range of those should be studied. These ranges appear only for types $\mathrm{C}$ and $\mathrm{D}$ as can be observed in the following figure (2).

Four different areas could be distinguished:

- The two blue ones indicate the voltage shift fluctuation range for the second and the third phases during a sag type $\mathrm{D}$.

- The two yellow ones indicate the voltage shift fluctuation range for the second and the third phases during a sag type $\mathrm{C}$.

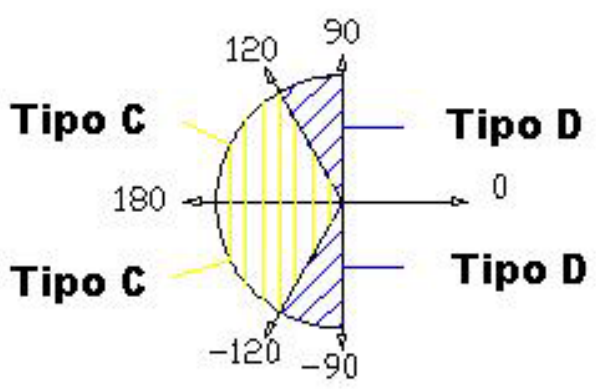

Fig. 7 Variation ranges for voltage shifts in sags $\mathrm{C}$ and $\mathrm{D}$

The rest of sags have not been considered since they occur in balanced conditions and only influence the voltage amplitude. In the fuzzification of the first voltage shift, three fuzzy sets are distinguished. From $-180^{\circ}$ to $0^{\circ}$, one representing $0^{\circ}$ and from $0^{\circ}$ to $180^{\circ}$.

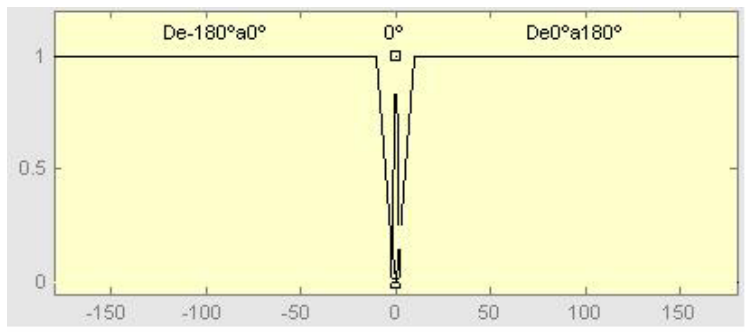

Fig. 8 Membership functions for the first voltage shift

The previous fuzzy sets are based in the expressions showed in Table I, where the first phase presents the same shift for all the cases. Regarding the voltage shift for the second phase, it exists three fuzzy sets, corresponding to following ranges: From $-180^{\circ}$ to $-120^{\circ}$, one representing $-120^{\circ}$ and from $-120^{\circ}$ to $-90^{\circ}$.

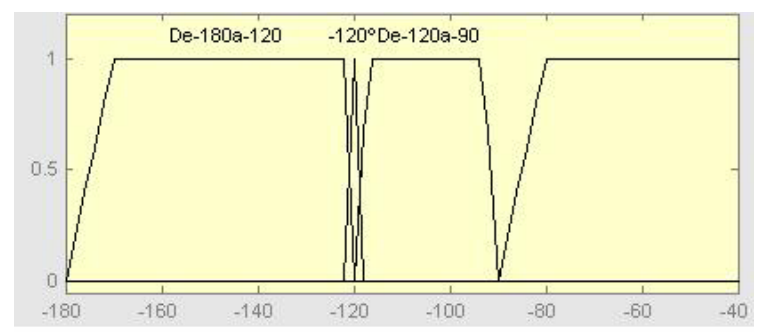

Fig. 9 Membership functions for the second voltage shift

Finally, the voltage shift for the third phase respond to the same division undertaken for the other cases.

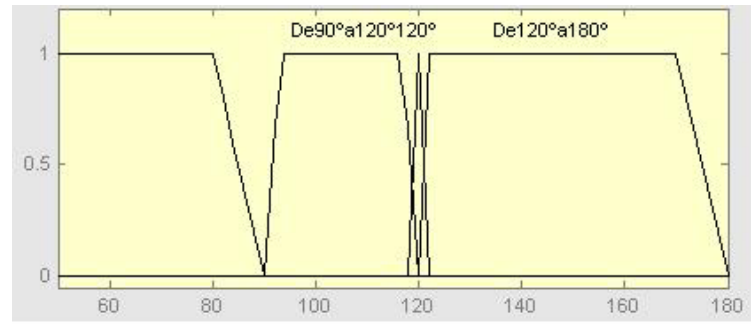

Fig. 10 Membership functions for the third voltage shift 


\section{Laboratory Setup}

Observing that the algorithm works well in the simulation, the practical implementation in a real measurement platform was undertaken. Thus, the previous matlab file (.fis) had to be converted to $\mathrm{C}$ code. The stand-alone $\mathrm{C}$ code fuzzy inference engine can read a FIS file and an input data file to perform fuzzy inference directly, or it can be embedded in other external applications.

The set-up consists of a programmable AC power source capable of generating distorted waveforms (different types of voltage sags), and an induction motor as load.

A DSP based measurement platform was inserted in this simple system. Their voltage readings were analyzed by means of the fuzzy logic, obtaining the type of the concrete sag generated.

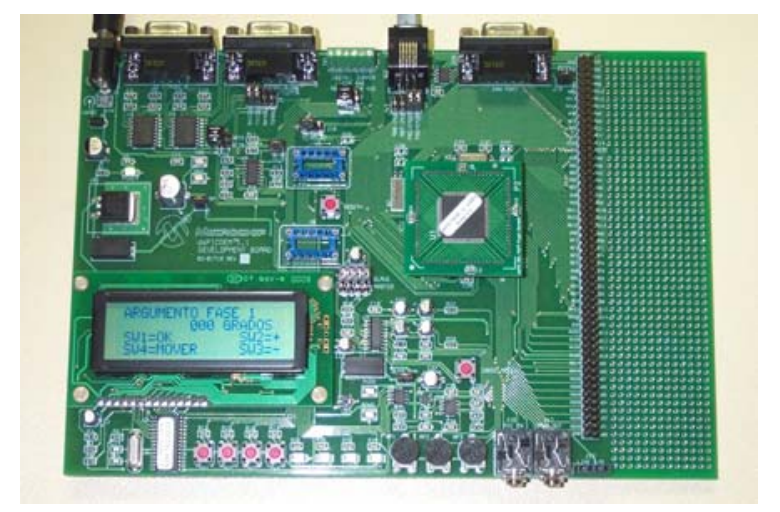

Fig. 11 dsPICDEM 1.1 Development Board

The measurement platform is based on a general purpose development board (dsPICDEM 1.1) which provides a low cost development tool based on a Digital Signal Controller (DSC) 16-bit architecture with high performance peripherals and powerful instruction set. It serves as an ideal prototyping tool to quickly develop and validate design requirements.

The board features an active development program loaded on the installed dsPIC30F6014A device. Several program functions are selectable via a menu system displayed on the LCD. Users can create a project, assemble and link the code, program and/or debug the code using Microchip's MPLAB ${ }^{\circledR}$ IDE Integrated Development Environment.

\section{Conclusions}

A fuzzy-expert system has been presented in this paper for detecting and classifying voltages sags. By means of a system of rules, one could characterize completely a sag. The four more common types proposed by Bollen's classification; A, B, C and D were considered. The inputs into the system are the voltage measurements resulting from the PCC.

The fuzzy algorithm was first tested by simulation with the interface of the Matlab Fuzzy Toolbox. Once the code run properly, it was translated into $\mathrm{C}$ code in order to be implemented in a DSP based measurement platform, obtaining a satisfactory result.

These concepts are easily programmable in current energy meters. In addition, the registration of this information can be very useful for invoicing purposes.

\section{Acknowledgement}

The authors would like to thank the support of the Spanish Government under the CICYT research project DPI2002-04416-C04-01.

\section{References}

[1] IEEE Project 1346 Working Group, Electric power system compatibility with industrial process equipment. Part 1. Voltage sags. IEEE Industrial and Commercial Power Systems Technical Conference, Irvine, CA, USA, May 1-5; 1994. p. 261-6.

[2] IEEE Std 1159-1995, IEEE recommended practice for monitoring electric power quality. New York: IEEE; 1995.

[3] Kezunovic M, Heydt G, Schilleci J, Lambert-Torres G, Key T, Kreis D. Intelligent system applications to power quality and substation automation. Panel Session at the IEEE Power Engineering Society Summer Meeting, Edmonton, Alberta, Canada, July 18-22, vol. 1.; 1999. p. 468-75.

[4] Kezunovic M, Liao Y. Use of intelligent techniques in the power quality assessment applications. International Conference of Intelligent Systems Applications to Power Systems, 1SAP, 2001. Budapest, Hungary, June 18-21 2001.

[5] Liao Y. Automated analysis of power quality data and transmission line fault location. $\mathrm{PhD}$ Dissertation. Texas A\&M University; 2000.

[6] M. Bollen, "Understanding power quality problems : voltage sags and interruptions".

[7] Yen J, Langari R. Fuzzy logic: intelligence, control, and information. Englewood Cliffs, NJ: PrenticeHall; 1999. 